\title{
Intramedullary compression device for proximal ulna fracture
}

Choon Chiet $\underline{\text { Hong}}^{1}$, MBBS, MRCSE, Fucai $\underline{\text { Han }}^{1}$, MBBS, MRCS, Joshua Decruz ${ }^{1}$, MD, Vinodhkumar Pannirselvam ${ }^{1}$, MBBS, MRCS, Diarmuid Murphy ${ }^{1}$, MB BCh BAO, FRCS

\begin{abstract}
Proximal ulna fractures account for $20 \%$ of all proximal forearm fractures. Many treatment options are available for such fractures, such as cast immobilisation, plate and screw fixation, tension band wiring and intramedullary screw fixation, depending on the fracture pattern. Due to the subcutaneous nature of the proximal forearm, it is vulnerable to open injuries over the dorsal aspect of the proximal ulna. This may in turn prove challenging, as it is critical to obtain adequate soft tissue coverage to reduce the risk of implant exposure and bony infections. We herein describe a patient with a Gustillo III-B open fracture of the proximal ulna, treated with minimally invasive intramedullary screw fixation using a 6.0-mm cannulated headless titanium compression screw (FusiFIX'M ${ }^{\mathrm{TM}}$, Péronnas, France).
\end{abstract}

Keywords: FusiFIX, intramedullary screw fixation, open fracture, proximal ulna fracture

\section{INTRODUCTION}

Proximal ulna fractures account for $20 \%$ of all proximal forearm fractures. ${ }^{(1)}$ There are many options available for the treatment of such fractures, such as cast immobilisation, plate and screw fixation, tension band wiring and intramedullary screw fixation, depending on the fracture pattern. ${ }^{(2-5)}$ Undisplaced proximal ulna fracture with intact extensor mechanism can be treated with cast immobilisation, while simple transverse fractures are best treated via tension band wiring and intramedullary screw fixation. The use of plate and screw fixation is more appropriate in comminuted fractures of the proximal ulna. Due to the subcutaneous nature of the proximal forearm, it is vulnerable to open injuries over the dorsal aspect of the proximal ulna. This may in turn prove challenging, as it is critical to obtain adequate soft tissue coverage to reduce the risk of implant exposure and bony infections. We herein describe a patient with a Gustillo III-B open fracture of the proximal ulna, treated with minimally invasive intramedullary screw fixation using a $6.0-\mathrm{mm}$ cannulated headless titanium compression screw.

\section{CASE REPORT}

A 43-year-old, right-hand-dominant construction worker presented with a Gustillo III-B open fracture of the right proximal ulna after a road traffic accident. The wound, which was located over the dorsal region of the proximal ulna, measured $15 \mathrm{~cm} \times$ $5 \mathrm{~cm}$. There was no neurological or vascular injury of the right upper limb, or any other injuries. Radiographic assessment showed an oblique proximal ulna fracture with some comminution at the fracture site and a radial head fracture involving less than $20 \%$ of the articular surface (Fig. 1). Intramuscular anti-tetanus toxoid and intravenous antibiotic were started. Due to the extent of the soft tissue injury, a spanning external fixator was applied across his elbow. The elbow wound was debrided and dressed via negative pressure wound therapy using vacuum-assisted closure (VAC) therapy ( $\mathrm{KCl}$, San Antonio, TX, USA). Postoperative radiographs showed satisfactory reduction and alignment (Fig. 2).

The patient underwent further wound debridement in the operating theatre to ensure a healthy wound bed before soft tissue coverage was done. VAC therapy was continued while waiting for bony fixation and wound coverage. Serial wound inspections showed a clean wound with granulation tissues and healthy skin edges. Tissue samples were negative for any bacterial growth.

We were unable to perform the standard plate and screw construct fixation for our patient in view of the concomitant large wound over the proximal ulna region, which may lead to a risk of implant exposure through the wound and subsequent infection. Hence, a decision was made to use an intramedullary device at the proximal ulna to prevent the risk and minimise further soft tissue disruption. The radial head fracture was treated conservatively because it involved less than $20 \%$ of the articular surface and the joint was stable when tested intraoperatively. Definite fixation was performed on Day 8 post-injury.

A 6.0-mm cannulated headless titanium compression screw (FusiFIX ${ }^{\mathrm{TM}}$, Péronnas, France) was used. Under direct visualisation of the fracture site through the wound, the fracture ends were reduced using pointed reduction clamps and confirmed via intraoperative fluoroscopy. A stab incision was made over the olecranon process, the triceps tendon was divided and a guidewire was inserted under fluoroscopic guidance. After the cortical bone was drilled, countersunk and measured, the FusiFIX ${ }^{\mathrm{TM}}$ 6.0-mm screw $(100 \mathrm{~mm})$ was inserted using a torque screwdriver until the screw was released from the prehensive system. Extra care was taken to ensure that the proximal thread was buried in the cortical bone. Final fluoroscopic assessment found the fracture reduction and fixation to be satisfactory. Subsequently, the triceps tendon was repaired and skin closure was performed with a non-absorbable suture. The wound defect

${ }^{1}$ University Orthopaedics, Hand and Reconstructive Microsurgery Cluster, National University Hospital, Singapore

Correspondence: Dr Hong Choon Chiet, Resident, Department of Orthopaedic Surgery, National University of Singapore, National University Health System, 1E Kent Ridge Road, Singapore 119228. choonchiet@gmail.com 

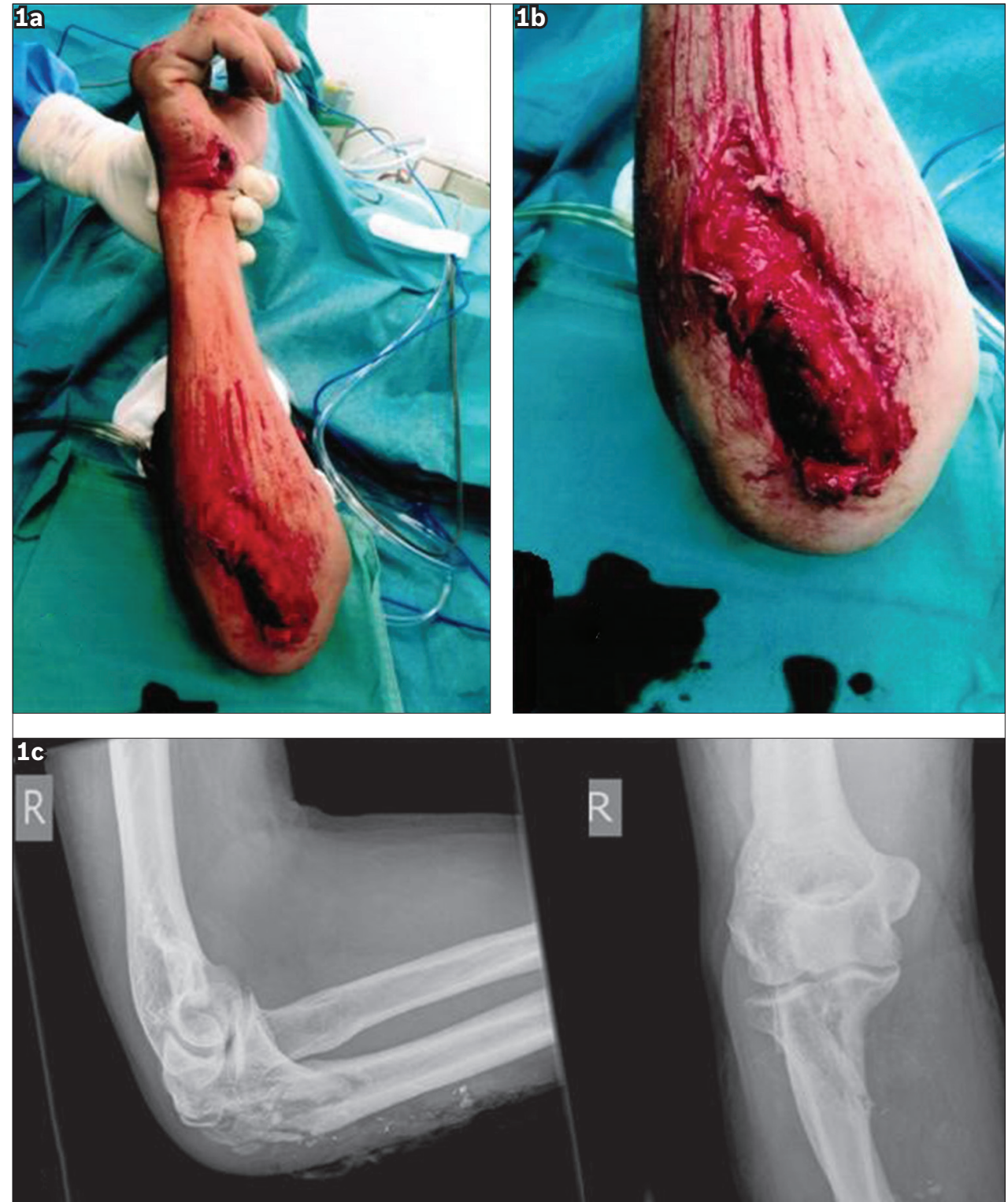

Fig. 1 (a \& b) Photographs show a large wound over the dorsal aspect of the proximal ulna. (c) Radiographs show the same elbow with proximal ulna fracture and soft tissue disruption over the proximal ulna region, consistent with the clinical presentation.

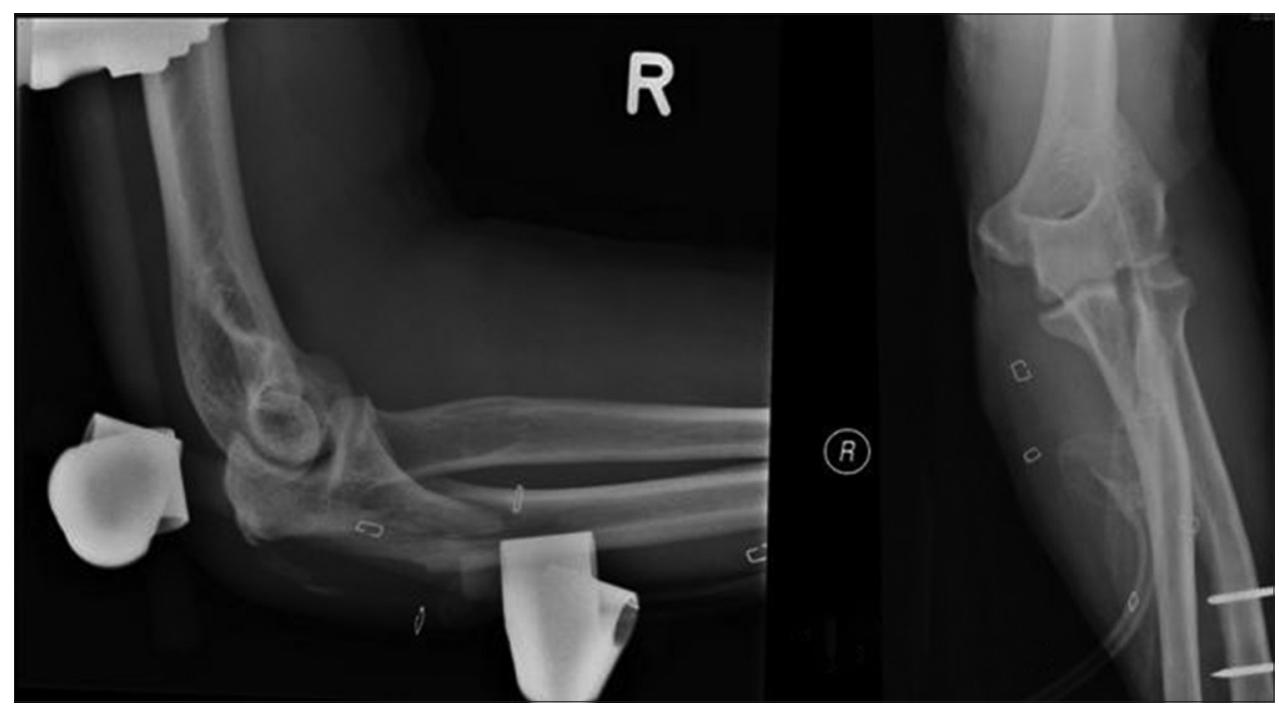

Fig. 2 Radiographs show the elbow after wound debridement, fracture reduction and external fixator application. Skin staples were used in applying vacuum-assisted closure therapy to the wound bed. 
over the proximal ulna was covered with split-thickness skin grafting (STSG) from the right medial thigh in the same setting. Intraoperative assessment showed a stable range of motion. An above elbow posterior plaster splint was applied to protect the osteosynthesis and graft site.

Postoperative radiographic review showed a well-compressed fracture site using the FusiFIX ${ }^{\mathrm{TM}}$ 6.0-mm screw. Wound inspection performed on postoperative Day 5 showed that the graft had fully taken. The patient was discharged with a universal arm sling and passive range of motion exercises. Three weeks postoperatively, the STSG had fully healed (Fig. 3). The patient was then started on active elbow range of motion exercises with assistance from the physiotherapist. Bony union was observed radiographically at 8-10 weeks and muscle strengthening exercises were commenced (Fig. 4). The patient regained a good elbow range of motion at $0-120$ degrees, supination of 80 degrees and pronation of 60 degrees. He was able to get back to work at four months postoperatively. At his latest review (one year postoperatively), the patient had resumed his active lifestyle.

\section{DISCUSSION}

Elbow fractures are commonly seen in orthopaedic practice and they account for $5 \%$ of all fractures. ${ }^{(2)}$ Most elbow fractures are of the proximal forearm and $20 \%$ of these are proximal ulna fractures. ${ }^{(1)}$ Open reduction and internal fixation is the standard treatment for displaced proximal ulna fractures. ${ }^{(3-5)}$ The surgical technique is dependent on a variety of factors, including patient factors, fracture pattern and mechanical stability of the osteosynthesis applied to stabilise the fracture. Treatment goals include anatomical reconstruction of the articular surface, restoration of joint stability, preservation of extensor mechanism and prevention of joint stiffness. ${ }^{(2,4)}$ Several treatment options for open reduction and internal fixation have been described, including tension band wiring, plate and screw fixation, triceps advancement after fragment excision and intramedullary screw fixation. Tension band wiring is designed to convert the tensile distraction force of the triceps into a dynamic compressive force at the articular surface and is suited for most simple transverse non-comminuted fractures. ${ }^{(5,6)}$ Plate and screw fixation is most commonly used for comminuted fractures in which tension band wiring is not feasible. It is also indicated for oblique fractures distal to the trochlear notch or fractures involving the coronoid process. ${ }^{(3,4,7)}$ Excision of the proximal fracture fragment and advancement of triceps may be used in elderly patients with osteoporotic bone or as a salvage procedure if internal fixation fails. ${ }^{(4,5)}$ Intramedullary screw fixation can be used for simple transverse non-comminuted fractures or fixation of olecranon osteotomy. ${ }^{(3-5)}$ All operative techniques should provide stable fixation to allow early motion, as loss of motion and joint stiffness are common after fractures of the elbow..$^{(2-5)}$

The FusiFIX ${ }^{\text {TM }}$ screw is a cannulated headless titanium screw with triple thread, which comes in $4.0 \mathrm{~mm}$ and $6.0 \mathrm{~mm}$ sizes. ${ }^{(8)}$ The screw is made up of three different segments with threads of different diameters that are designed to provide a greater

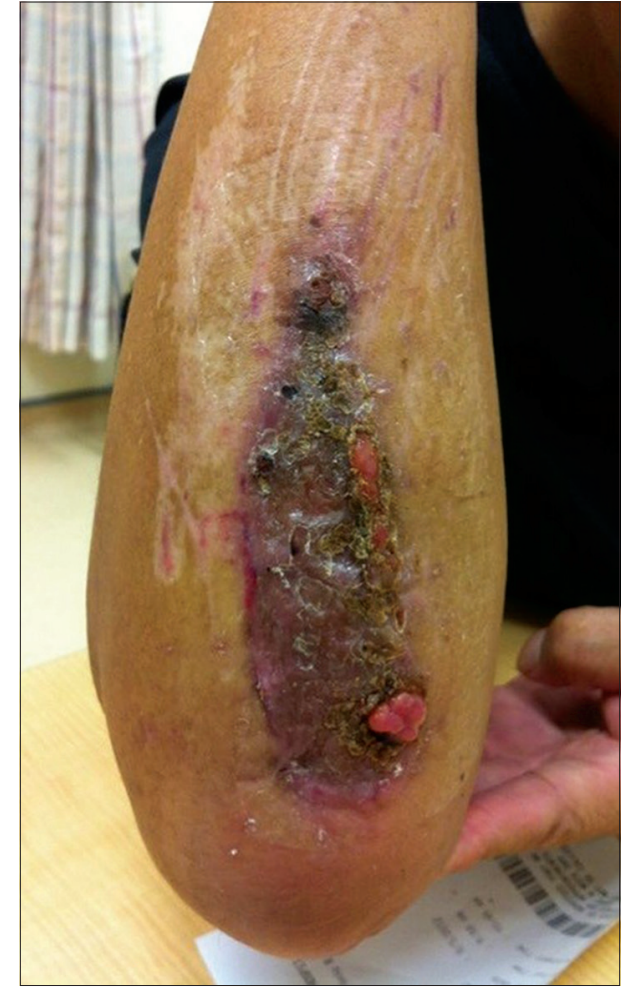

Fig. 3 Photograph shows the dorsal wound healing at three weeks postoperatively.

compressive force. It is completely threaded to enable better maintenance of cyclical loading during the healing period. The FusiFIX ${ }^{\mathrm{TM}}$ screw is commonly used for middle and rear foot osteotomies and arthrodesis. In this report, we described a patient with a Gustillo III-B open fracture of the proximal ulna who was treated with minimally invasive intramedullary screw fixation using the 6.0-mm FusiFIX ${ }^{\mathrm{TM}}$ screw.

Wadsworth reported that the use of partially threaded intramedullary screw with or without a washer in displaced olecranon fractures or osteotomy fixation had good results, with $100 \%$ union in six patients and no complications. In addition, the patients were reported to have an excellent range of motion for up to an average of 1 year follow-up postoperatively and nearly equivalent range of motion to the contralateral elbow. ${ }^{(9)}$ Using an $\mathrm{AO}$ (Arbeitsgemeinschaft für osteosynthesefragen) $6.5-\mathrm{mm}$ cancellous bone screw, Johnson et al demonstrated good results with 28 patients, with $67 \%$ of patients acquiring full motion by postoperative Week 9. ${ }^{(10)}$ Murphy et al demonstrated the use of screw fixation in displaced olecranon fracture, resulting in superior outcome for pain, function and range of motion compared to tension band wiring. ${ }^{(11)}$ Molloy et al reported in a cadaveric study that intramedullary nail fixation of olecranon fracture is significantly stronger and stiffer compared to tension band wiring. ${ }^{(12)}$ Our patient underwent an intramedullary compression device fixation using the FusiFIX ${ }^{\mathrm{TM}}$ screw via a minimally invasive incision over the olecranon process, avoiding the dorsal wound. Our aim was to achieve and maintain a stable fracture reduction without further soft tissue disruption at the fracture site. This was to facilitate early functional rehabilitation as well as avoid potential implant exposure that may lead to soft tissue and bony infections. Despite 


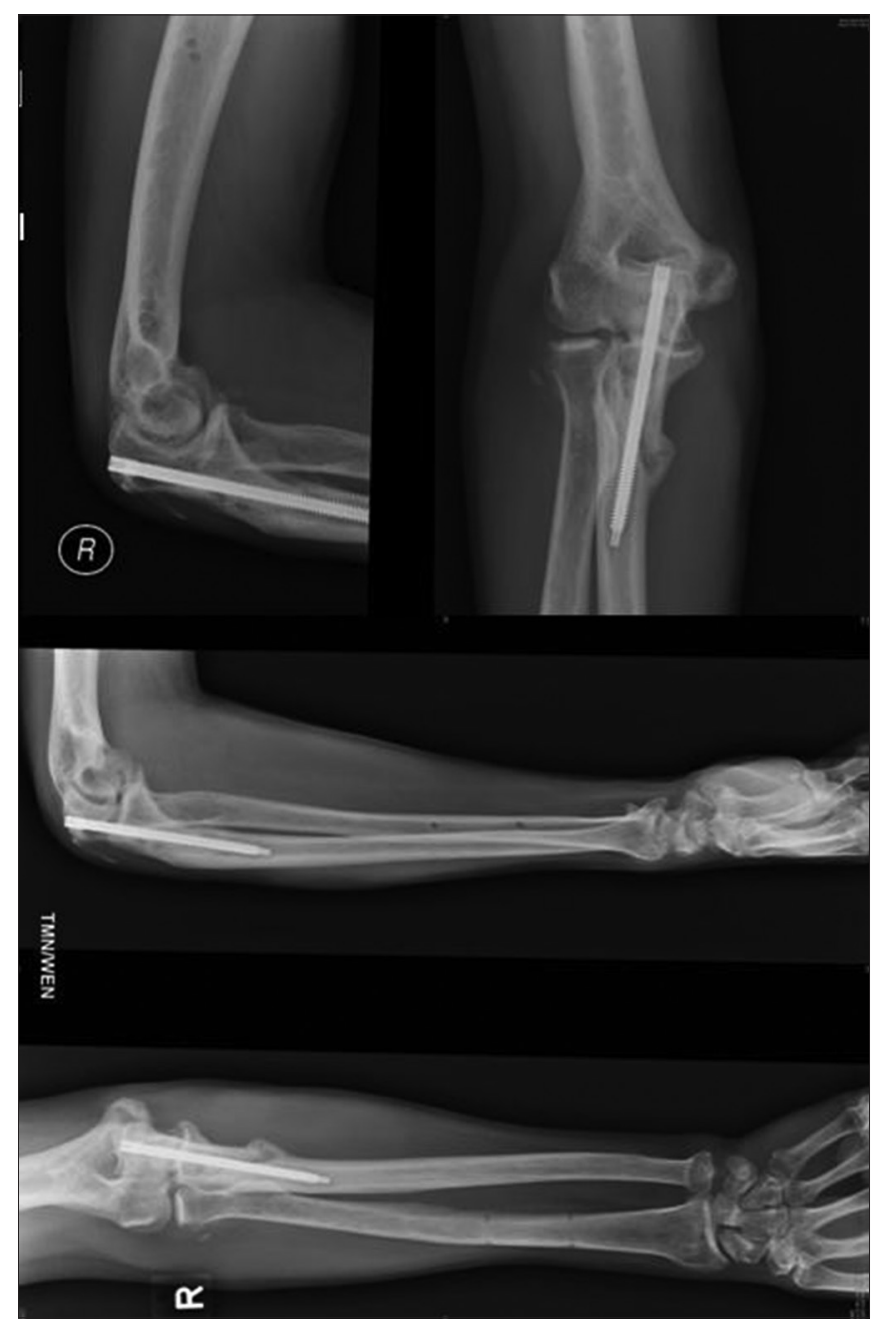

Fig. 4 Radiographs show the internal fixation of the proximal ulna fracture with the FusiFIX'M screw.

the oblique fracture pattern of the fracture, this technique obviates the need for further periosteal stripping, which could affect fracture union. It also reduces the risks of direct implant exposure through the wound site in the event of wound breakdown or STSG failure. We believe that intramedullary compression device fixation in this case also allows for earlier mobilisation without the surrounding soft tissue irritation caused by plate fixation or tension band wiring, and obviates the need for implant removal. In addition, the compressive force provided by the FusiFIX ${ }^{\mathrm{TM}}$ screw would allow adequate compression for primary bone healing.

The FusiFIX ${ }^{\mathrm{TM}}$ screw has a cylindrical shape, and the conical core of the screw enables the distal part of the screw's thread to penetrate the bone more quickly than the proximal part of the thread. These features enable progressive compression of the bone fragments during penetration of the screw and maintenance during the insertion. It is also self-tapping and cannulated, which facilitate easier insertion and reduce operating time. The absence of the screw head allows total sinking of the screw into the bone and prevents the risk of loosening or irritation of the surrounding soft tissue. Since the fixation was designed for use in cancellous bone, its use in our patient would create a compressive force at the proximal ulna, which is made up of a significant portion of cancellous bone. Its design would also allow the screw to function as an intramedullary device and splint the fragments immediately distal to the olecranon, allowing greater stability and facilitating earlier return to motion. Our patient was able to perform active elbow range of motion bearing exercise by postoperative Week 3, once the STSG had fully healed. He also regained almost equivalent range of motion to his contralateral elbow. This concurs with the findings reported by Wadsworth and Johnson et al. ${ }^{(9,10)}$

Proximal ulna fractures can occur in isolation or with more complex injuries of the elbow. Undisplaced fractures with intact extensor mechanism can be treated conservatively, while open reduction and internal fixation is recommended for displaced fractures. There are multiple fixation options available for use depending on the fracture pattern, with each having its own strengths and weaknesses. ${ }^{(3,4)}$ In this report, we have highlighted a unique presentation of an open proximal ulna fracture with a large wound over the dorsal aspect of the proximal ulna, making fixation options limited. We elected to use an intramedullary device in our patient to avoid potential risks of implant exposure through the wound and to minimise soft tissue disruption. We opine that this technique allows for a stable fixation, which will enable early functional rehabilitation. To the best of our knowledge, this report is the first to describe the novel use of the intramedullary compression device, the FusiFIX ${ }^{\mathrm{TM}}$ screw, in the fixation of an open proximal ulna fracture with a large dorsal wound.

\section{REFERENCES}

1. Court-Brown CM, Caesar B. Epidemiology of adult fractures: A review. Injury 2006; 37:691-7

2. Cabanela ME, Morrey BF. Fractures of the proximal ulna and olecranon. In: Morrey BF, eds. The Elbow and Its Disorders, 2nd ed. Philadelphia: WB Saunders, 1993: 405-28.

3. Baecher N, Edwards S. Olecranon fractures. J Hand Surg Am 2013; 38:593604.

4. Hak DJ, Golladay GJ. Olecranon fractures: treatment options. J Am Acad Orthop Surg 2000; 8:266-75.

5. Morrey BF. Current concepts in the treatment of fractures of the radial head, the olecranon, and the coronoid. Instr Course Lect 1995; 44:175-85.

6. Müller ME, Allgöwer M, Schneider R, Willenegger $H$, eds. Manual of Internal Fixation: Techniques Recommended by the AO-ASIF Group, 3rd ed. Berlin: Springer-Verlag, 1991.

7. Schatzker J. Fractures of the olecranon. In: Schatzker J, Tile M, eds. The Rationale of Operative Fracture Care. Berlin: Springer-Verlag, 1987: 89-95.

8. FusiFIX ${ }^{\mathrm{TM}} 4.0$ \& $6.0 \mathrm{~mm}$. In: Small Bone Innovations, Inc [Online]. Available at: www.totalsmallbone.com/uk/products/foot_ankle/fusifix. php4. Accessed February 6, 2015.

9. Wadsworth TG. Screw fixation of the olecranon after fracture or osteotomy. Clin Orthop Relat Res 1976; 119:197-201.

10. Johnson RP, Roetker A, Schwab JP. Olecranon fractures treated with AO screw and tension bands. Orthopedics 1986; 9:66-8.

11. Murphy DF, Greene WB, Dameron TB Jr. Displaced olecranon fractures in adults. Clinical evaluation. Clin Orthop Relat Res 1987; 224:215-23.

12. Molloy S, Jasper LE, Elliott DS, Brumback RJ, Belkoff SM. Biomechanical evaluation of intramedullary nail versus tension band fixation for transverse olecranon fractures. J Orthop Trauma 2004; 18:170-4. 